\title{
Microstructural Analysis of 5XXX Series Aluminum Alloys Modified for Optimal Strength and Corrosion Resistance
}

\author{
Kinga A. Unocic, * Michael J. Mills, * Glenn S. Daehn, * and Paul Kobe** \\ * Department of Materials Science and Engineering, The Ohio State University, Columbus, $\mathrm{OH}$ \\ ** Pechiney Rolled Products, Ravenswood, WV
}

\begin{abstract}
Intergranular corrosion is a significant concern for Al-Mg alloys when subjected to a salt-water media. To address this, the standard AA5083 was modified in an attempt to improve corrosion resistance. STEM and EFTEM imaging was performed to analyze microstructural features that cause this behavior. Corrosion was attributed to a network of Mg-rich grain boundary precipitates in the standard alloy. Alloying with $\mathrm{Cu}$ and $\mathrm{Zn}$ was found to suppress grain boundary precipitation and thus improve corrosion resistance.
\end{abstract}

\section{Introduction}

$5 x x x$ series aluminum alloys with additions of magnesium greater than $3.0 \%$ were reported to be more susceptible to this form of corrosion when compared to that of the standard alloy composition.[1] Preferential corrosion sites along grain boundaries are linked to the segregation of magnesium and other precipitating phases such $\beta, \tau$ and $s$ phases, of which are anodic relative to the aluminum matrix.[2]

The purpose of this study was to modify the microstructure of standard 5083 aluminum alloys for increased intergranular corrosion resistance. An in depth microstructural characterization was performed using STEM imaging and EFTEM mapping to image and identify local phases and phase segregation along grain boundaries. Additionally, compositional line profiling was performed to determine the extent of this local elemental and phase segregation. TEM characterization results therefore provided the necessary link between detrimental corrosive phases that were responsible for the experimentally observed intergranular corrosion behavior.

\section{Materials and Experimental Procedure}

More than twenty modified AA5083 Pechiney alloys underwent corrosion testing following ASTM-G67 standard testing procedures. The prime differences between these alloys are the varying levels of copper, zinc, magnesium, silicon, manganese, titanium and iron relative to the standard AA5083 composition. The alloys were hot rolled with a high percentage of reduction in thickness followed by small amount of cold work. Sensitization heat treatments were subsequently applied to evaluate the precipitation of undesirable phases. Microstructural characterization was performed using a FEI Tecnai TF20 using STEM imaging, x-ray microanalysis and EFTEM mapping. TEM foils were prepared by electropolishing in an electrolyte containing $25 \%$ Nitric Acid and $75 \%$ Methanol at $-32^{\circ} \mathrm{C} / 10 \mathrm{~V}$.

\section{Results and Discussion}

Two alloys were initially chosen for microstructural characterization since they possessed extremely different corrosive behaviors. Fig. 1a presents analysis of the standard alloy sensitized for $20 \mathrm{~h}$ at $200^{\circ} \mathrm{C}$. As can be seen, the grain boundary is rich in a network of precipitates. EDX 
line profiling (Fig. 1b) shows an enrichment of Mg. This gives conformation that during the sensitization treatment $\mathrm{Mg}$ rich precipitates form along the boundaries; however, further analysis is needed to precisely identify the nature of the precipitate. The modified alloy, which performed better in the terms of corrosion resistance, was observed to have no continuous network of precipitates along grain boundaries. (Fig. 1c,d)

\section{Summary}

In conclusion, the results from intergranular corrosion testing showed that the standard alloy was more susceptible to intergranular corrosion when compared to samples with $\mathrm{Cu}$ and $\mathrm{Zn}$ additions. The addition of a balanced amount of zinc or copper increases the resistance to intergranular corrosion by modifying the type and location of precipitates that form during sensitization treatments. STEM imaging, coupled with EDX line profiling, and EFTEM imaging was used to identify the type of precipitates that were responsible for this behavior.

\section{References}

[1] M.O. Speidel, M.V. Hyatt, in: M.G. Fontana, R.W. Staehle (Eds.), Advances in Corrosion Science and Technology, Plenum Press, New York, 1972.

[2] M.C. Carroll, et al, Scripta Materialia, 42, (1999), 335-340.
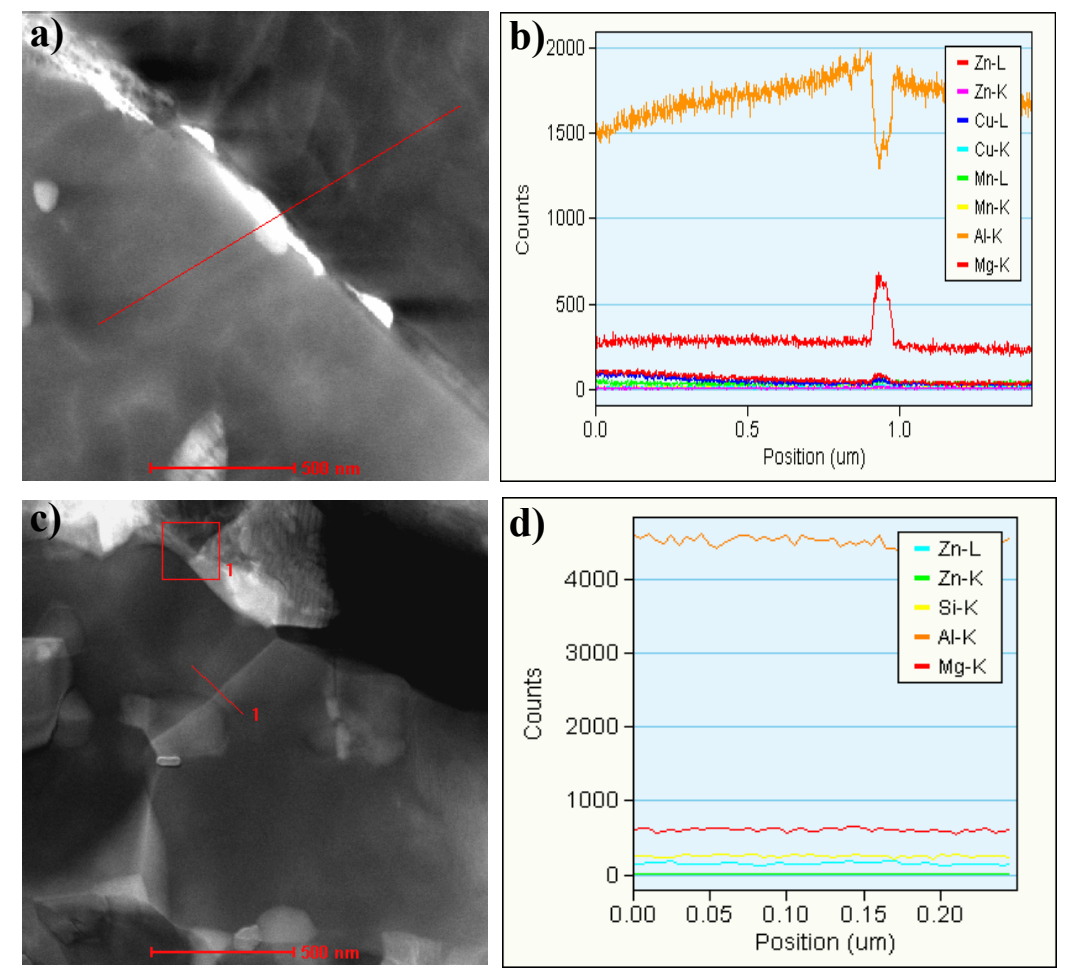

FIG. 1. a) Dark field STEM image of the standard alloy composition showing grain boundary precipitates with b) corresponding EDX line profile analysis c) Dark field STEM image from the modified AA5083 with d) corresponding EDX line profile analysis. 